\title{
Dependence of the cloud size on interactions for a trapped degenerate ultracold plasma
}

\author{
S. Sevinçli, M.Ö. Oktel, B. Tanatar* \\ Bilkent University, Department of Physics, 06800 Bilkent, Ankara, Turkey \\ Received 24 August 2005; accepted 3 October 2005 \\ Available online 12 October 2005 \\ Communicated by V.M. Agranovich
}

\begin{abstract}
We consider an ultracold plasma that has bosonic ions, at zero temperature. Assuming that ions are trapped by a harmonic trap, we calculate the size of the cloud for both screened and bare Coulomb interactions. Our results indicate that if clouds containing around $10^{4}$ ions are confined with a trapping frequency of $10 \mathrm{kHz}$, stable radius is $15 \mu \mathrm{m}$ for a fully screened two-component plasma while the radius increases to 2 mm for a one component plasma.

(C) 2005 Elsevier B.V. All rights reserved.
\end{abstract}

\section{Introduction}

The advances in trapping and cooling methods for neutral atoms have led to remarkable experiments where quantum mechanical nature of bosonic and fermionic particles can be studied at very low temperatures. The systems where the temperatures are low enough for quantum mechanical effects to be dominant are called ultracold systems. After the study of BoseEinstein condensation, using neutral bosonic atoms [1], study of ultracold atoms have expanded into the investigation of fermion systems [2] and ultracold molecule production via Feshbach resonances [3]. All these experiments have the common property that the investigated particles are neutral.

Another new direction in ultracold systems is the creation of an ultracold plasma [4]. An ultracold plasma is created by rapid ionization from an ultracold gas, and so far its investigation can only be done in a dynamical state. In current experiments, after rapid ionization, a fraction of the electrons leave the cloud, while the remaining cloud has a central region which is a neutral two component plasma [5-12]. The electron pressure inside the plasma causes rapid expansion of the cloud, limiting the observation time to hundreds of microseconds and complicating the

\footnotetext{
* Corresponding author.

E-mail address: tanatar@fen.bilkent.edu.tr (B. Tanatar).
}

investigation of the properties of the ultracold plasma. Although the dynamical properties have yielded interesting phenomena, creation of a stable ultracold plasma will be interesting both from a basic science point of view and may lead to new technological advances in fields ranging from precision measurements to quantum computation.

For an ultracold plasma, the electron Coulomb coupling parameter $\Gamma_{e} \simeq e^{2} n^{1 / 3} / k_{B} T$, which measures the ratio of average interaction energy between electrons to their thermal energy is close to unity [5-12]. The regime where this parameter is larger than unity is unexplored and proposes many fundamental questions. Especially when quantum mechanical correlations between the constituent particles are taken into account, one can expect many new phenomena to emerge. As examples for the fundamental questions about this system, one can ask "What is the nature of plasma oscillations when particles are strongly interacting and quantum degenerate?" or "Can electrons and ions coherently combine and dissociate, leading to natural atom-plasma oscillations?". Before attempting to tackle such questions, in this Letter, we consider a simple model of quantum degenerate plasma where the ions form a Bose condensate, and understand the dependence of the size of such a cloud on interactions.

So far, in ultracold plasma experiments, plasma is produced by rapid ionization from a cold gas trapped in a magneto-optical trap [5-12]. In the rapid ionization process, most of the energy 
is absorbed by the electrons, leaving the ion cloud at approximately same temperature as the original atom cloud. In principle electron temperature can also be controlled by tuning the excitation frequency very close to ionization threshold. Also by exciting atoms to Rydberg states, plasma formation through collisions has been demonstrated [13-18]. Initial temperature of the atomic cloud can be made very small, for example, Rydberg atom and ultracold plasma formation from a Bose Einstein condensate has been demonstrated [19].

Although stable trapping of ultracold plasma has not been demonstrated, there is no fundamental physical reason against construction of such a trap. One can envision two types of traps, those that couple the electric charge of the ions, such as the current ion traps; or those that couple to the electric dipole moment of the ions, similar to the optical traps used in cold atom experiments [20]. If an ion trap, such as a Penning trap [21,22] is used ions in the plasma will be trapped, while the electrons will be antitrapped and lost from the plasma. Such an ion trap has been used to look at crystallization and structural phase transitions of two-dimensional one component plasma at low densities [23]. In principle, such an ion trap can be used to trap the ions in an ultracold plasma, forming a one component, strongly coupled plasma at high densities. Another, and perhaps more interesting trap, would be an optical trap that couples to the excited states of ions, such a trap will not be directly affected by the free charges of electrons and ions as it would operate at much higher frequencies compared to the plasma frequencies in the cloud. Such a trap could be used to trap the ions while electrons will be trapped by the charge of the ion cloud as in the current experiments [5-12].

Expecting advances in trapping technology, we assume that a quadratic trap for the ions have been set up and the cloud has been stabilized. As ion traps and neutral atom traps have improved drastically over the last decade, we believe such a trap can be realized in the near future. We calculate the size of the cloud in two situations, first we assume that there is a constant density of electrons in the cloud giving rise to Thomas-Fermi screening [24] of the ion-ion interactions. Using a Gaussian variational wavefunction, we find the dependence of the cloud size on electron density. In the second case, we assume that all the electrons escape from the trap and leave a charged Bose gas and once again calculate the size of the cloud. A detailed calculation of the electron and ion densities, along with densities for vortex situations will be reported in a subsequent paper.

\section{Ultracold plasma}

We describe the ion cloud as a Bose condensed system within the mean-field approximation. The ground state energy at zero temperature is given by the Gross-Pitaevskii energy functional

$$
\begin{aligned}
E= & \int d \mathbf{r}_{\mathbf{1}}\left\{\frac{\hbar^{2}}{2 m}\left|\nabla \psi\left(\mathbf{r}_{\mathbf{1}}\right)\right|^{2}+\frac{1}{2} m \omega^{2} r_{1}^{2}\left|\psi\left(\mathbf{r}_{\mathbf{1}}\right)\right|^{2}\right. \\
& \left.+\int d \mathbf{r}_{\mathbf{2}}\left|\psi\left(\mathbf{r}_{\mathbf{1}}\right)\right|^{2}\left|\psi\left(\mathbf{r}_{\mathbf{2}}\right)\right|^{2} U\left(\mathbf{r}_{\mathbf{1}}-\mathbf{r}_{\mathbf{2}}\right)\right\},
\end{aligned}
$$

where $U(\mathbf{r})=\left(Z^{2} e^{2}\right) \mathrm{e}^{-\mu \mathbf{r}} / \mathbf{r}$ is the Yukawa potential between the ions which are assumed to be in an isotropic harmonic oscillator potential $m \omega^{2} \mathbf{r}^{2} / 2$. $U(\mathbf{r})$ models the screened Coulomb interaction between the ions through the screening parameter $\mu . \psi(\mathbf{r})$ is the ion condensate wavefunction. We introduce dimensionless units by making the following transformation: (i) $\mathbf{r} \rightarrow l \mathbf{r}$, where $l=\sqrt{\hbar / m \omega}$ is oscillator length, and (ii) the energy is measured in units of $\hbar \omega$. Using the dimensionless quantities, we can rewrite the energy functional as

$$
\begin{aligned}
\frac{E}{\hbar \omega}= & \frac{1}{2} \int d \mathbf{r}_{1}\left\{\left|\nabla \psi\left(\mathbf{r}_{1}\right)\right|^{2}+r_{1}^{2}\left|\psi\left(\mathbf{r}_{1}\right)\right|^{2}\right\} \\
& +\gamma \iint d \mathbf{r}_{1} d \mathbf{r}_{2} \frac{\left|\psi\left(\mathbf{r}_{1}\right)\right|^{2}\left|\psi\left(\mathbf{r}_{2}\right)\right|^{2} e^{-\mu\left|\mathbf{r}_{1}-\mathbf{r}_{2}\right|}}{\left|\mathbf{r}_{1}-\mathbf{r}_{2}\right|},
\end{aligned}
$$

where

$\gamma=\frac{\left(Z^{2} e^{2}\right)}{\hbar} \sqrt{\frac{m}{\hbar \omega}}$

is a dimensionless coupling strength for the interaction between the ions. It measures the ratio of the interaction energy between the ions to their trapping energy. Note that this parameter is different from the usual Coulomb coupling parameter which is used in plasma physics.

We use the variational principle to obtain the condensate wavefunction that minimizes the Gross-Pitaevskii functional. For simplicity, we choose a Gaussian trial wave function,

$\psi(r)=\left[N\left(\frac{2 \alpha}{\pi}\right)^{3 / 2}\right]^{1 / 2} \mathrm{e}^{-\alpha r^{2}}$,

with a variational parameter $\alpha$. Note that $\psi$ is normalized. The kinetic and external potential energy terms in the energy functional are easily calculated to be $3 N \alpha / 2$ and $3 N / 8 \alpha$, respectively. To calculate the interaction term we go to the center-ofmass coordinate system,

$\mathbf{R}=\frac{\mathbf{r}_{1}+\mathbf{r}_{2}}{2} \quad$ and $\quad \mathbf{r}=\mathbf{r}_{1}-\mathbf{r}_{2}$,
$\mathbf{r}_{\mathbf{1}}=\mathbf{R}+\frac{1}{2} \mathbf{r} \quad$ and $\quad \mathbf{r}_{2}=\mathbf{R}-\frac{1}{2} \mathbf{r}$.

The interaction energy term becomes

$$
\begin{aligned}
E_{I} & =\gamma N^{2}\left(\frac{2 \alpha}{\pi}\right)^{3} \iint d \mathbf{R} d \mathbf{r} \frac{\mathrm{e}^{-4 \alpha R^{2}} \mathrm{e}^{-\left(\alpha r^{2}+\mu r\right)}}{r} \\
& =\frac{2 \gamma N^{2}}{\sqrt{\pi}} \alpha^{1 / 2}-\gamma N^{2} \mu \mathrm{e}^{\frac{\mu^{2}}{4 \alpha}} \operatorname{erfc}\left(\frac{\mu}{2 \sqrt{\alpha}}\right) .
\end{aligned}
$$

Finally, the total energy reads

$$
\frac{E}{N \hbar \omega}=\frac{3}{2} \alpha+\frac{3}{8 \alpha}+\frac{2 N \gamma}{\sqrt{\pi}} \alpha^{1 / 2}-\gamma N \mu \mathrm{e}^{\frac{\mu^{2}}{4 \alpha}} \operatorname{erfc}\left(\frac{\mu}{2 \sqrt{\alpha}}\right) .
$$

Minimizing the total energy with respect to $\alpha$, we get

$$
\begin{gathered}
\frac{3}{2}-\frac{3}{8} \alpha^{-2}+\frac{N \gamma}{\sqrt{\pi}} \alpha^{-1 / 2}-\frac{N \gamma \mu^{2}}{2 \sqrt{\pi}} \alpha^{-3 / 2} \\
+\frac{N \gamma \mu^{3}}{4} \mathrm{e}^{\frac{\mu^{2}}{4 \alpha}} \operatorname{erfc}\left(\frac{\mu}{2 \sqrt{\alpha}}\right) \alpha^{-2}=0 .
\end{gathered}
$$




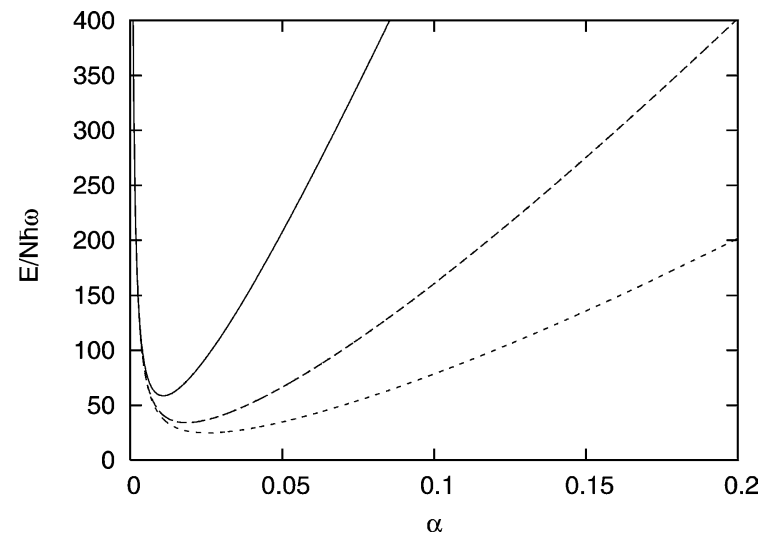

Fig. 1. Total energy per particle in units of $\hbar \omega$ as a function of the variational parameter $\alpha$ for $N=10^{4}$ atoms and different screening parameters for the Yukawa potential. Solid, dashed, and dotted lines are for $\mu=1,2$, and 3, respectively. The Coulomb coupling parameter is $\gamma=1$.

Although the Coulomb coupling parameter $\gamma$ is considered to be of the order of unity in the literature [25] for the charged Bose gas, realistic calculations of $\gamma$ with experimental parameters [26] give a value of the order of $10^{8}$. As an illustration we first give the variational parameter $\alpha$ dependence of the total energy per particle in units of $\hbar \omega$ for $\gamma=1$ and $N=10^{4}$ atoms in Fig. 1. One can observe the minimum of the energy for various screening parameters in the figure. We shall address the more realistic case of large values of $\gamma$ shortly.

The screening parameter $\mu$ in the screened Coulomb potential can be defined in terms of the density $n_{0}$ within the Thomas-Fermi (TF) approximation. The TF approximation consists in assuming that a local internal chemical potential can be defined as a function of the electron concentration at that point. In the TF theory, the electron density is represented locally as a free particle system and the chemical potential is independent of position. Then, Thomas-Fermi screening length $1 / \mu$ is defined as

$\mu^{2}=4\left(\frac{3}{\pi}\right)^{1 / 3} \frac{n_{0}^{1 / 3}}{a_{B}}$,

where $a_{B}$ is the Bohr radius. The density at the center can be defined by means of the variational parameter $\alpha$

$n_{0}=\frac{N}{\frac{4 \pi}{3} \alpha^{-3 / 2}}$.

Then, one can write the screening parameter as a function of the variational parameter $\alpha$

$\mu=\beta N^{1 / 6} \alpha^{1 / 4}$

where we have introduced a dimensionless quantity $\beta=$ $(12 / \pi)^{1 / 3}\left(l / a_{B}\right)^{1 / 2}$. Using the TF value of $\mu$ in Eq. (8), the total energy per particle becomes

$$
\begin{aligned}
\frac{E}{N \hbar \omega}= & \frac{3}{2} \alpha+\frac{3}{8 \alpha}+\frac{2 N \gamma}{\sqrt{\pi}} \alpha^{1 / 2} \\
& -\gamma N^{7 / 6} \beta \mathrm{e}^{\frac{\beta^{2} N^{1 / 3}}{4 \alpha^{1 / 2}}} \operatorname{erfc}\left(\frac{\beta N^{1 / 6}}{2 \alpha^{1 / 4}}\right) \alpha^{1 / 4} .
\end{aligned}
$$

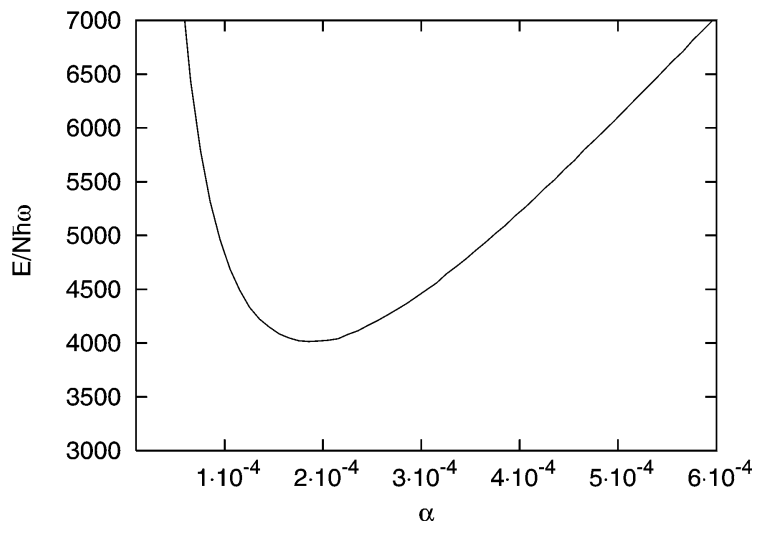

Fig. 2. Total energy per particle in units of $\hbar \omega$ as a function of the variational parameter $\alpha$ for $N=10^{4}$ atoms for Yukawa potential. The Coulomb coupling parameter is $\gamma=10^{8}$.

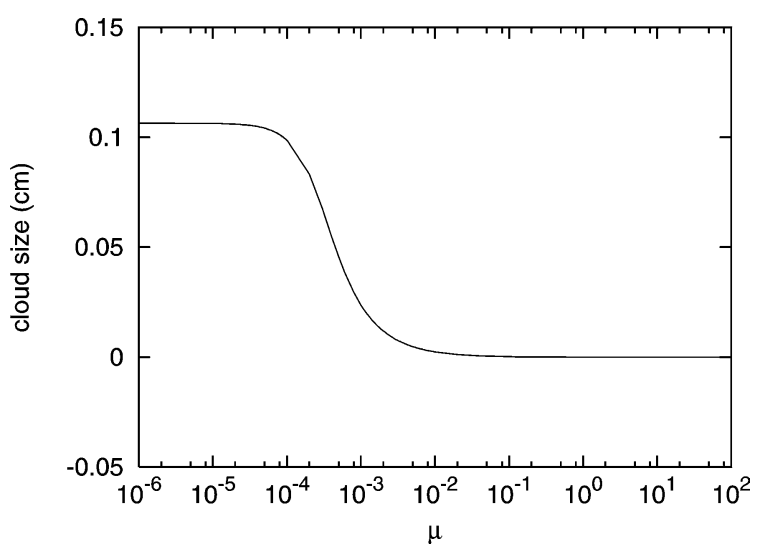

Fig. 3. Cloud size $1 / \sqrt{\alpha}$ as a function of the screening parameter $\mu$ for $N=10^{4}$ atoms. Coulomb coupling parameter is $10^{8}$. Two limits of the $\mu$ dependence is shown. For small values of the screening parameter $\mu$, the cloud size decreases since the screening reduces the range of Coulomb potential. In the opposite limit, as $\mu$ goes to zero the value of the cloud size corresponds to that of the bare Coulomb potential, i.e., charged Bose gas.

Minimizing the energy with respect to $\alpha$, we get

$$
\begin{aligned}
\frac{3}{2} & -\frac{3}{8} \alpha^{-2}+\frac{N \gamma}{\sqrt{\pi}} \alpha^{-1 / 2}-\frac{N^{4 / 3} \gamma \beta^{2}}{4 \sqrt{\pi} \alpha} \\
& -\frac{N^{7 / 6} \gamma \beta}{4 \alpha^{3 / 4}} \mathrm{e}^{\frac{\beta^{2} N^{1 / 3}}{4 \alpha^{1 / 2}}} \operatorname{erfc}\left(\frac{\beta N^{1 / 6}}{2 \alpha^{1 / 4}}\right) \\
+ & \frac{N^{3 / 2} \gamma \beta^{3}}{8 \alpha^{5 / 4}} \mathrm{e}^{\frac{\beta^{2} N^{1 / 3}}{4 \alpha^{1 / 2}}} \operatorname{erfc}\left(\frac{\beta N^{1 / 6}}{2 \alpha^{1 / 4}}\right)=0 .
\end{aligned}
$$

Fig. 2 shows the dependence of the total energy per particle in units of $\hbar \omega$ on $\alpha$, which is the inverse square of the cloud size, for $N=10^{4}$ and the Coulomb coupling parameter $\gamma=10^{8}$. It can be seen that there is still a minimum of energy for the realistic parameters. We solve Eq. (14) numerically to find the variational parameter $\alpha$ for various values of the parameters $N, \mu$ and $\gamma$. Our estimate of the cloud size relies on the experimental parameters of Chen et al. [26] who had $N=10^{4}$ atoms. Thus, for $10^{4}$ atoms we obtain the cloud size for the screened Coulomb interaction to be $\sim 15 \mu \mathrm{m}$ for which the trap frequency is approximately $10^{4} \mathrm{~Hz}$. In Fig. 3 the dependence of the size of a Bose condensed ionic cloud on 
the electron density which is obtained using the Thomas-Fermi screening picture is shown. Two limiting behaviors are evident. For the large values of the screening parameter $\mu$, the cloud size, $1 / \sqrt{\alpha}$, decreases as expected, since the screening reduces the range of the Coulomb potential. As the screening parameter $\mu$ goes to zero the value of the cloud size corresponds to that of bare Coulomb potential case, i.e., charged Bose gas.

\section{Charged bosons confined in a harmonic trap}

We now consider the situation of a system composed of $N$ identical bosons interacting via the repulsive Coulomb interaction $Z^{2} e^{2} / r$ that are confined in an isotropic harmonic trap. As in the case of ultracold plasma of ions interacting via the Yukawa potential, we use the Gross-Pitaevskii functional to describe the ground state properties. In dimensionless units introduced previously, the Gross-Pitaevskii energy functional is given by

$$
\begin{aligned}
\frac{E}{\hbar \omega}= & \frac{1}{2} \int d \mathbf{r}_{1}\left\{\left|\nabla \psi\left(\mathbf{r}_{1}\right)\right|^{2}+r_{1}^{2}\left|\psi\left(\mathbf{r}_{1}\right)\right|^{2}\right\} \\
& +\gamma \iint d \mathbf{r}_{1} d \mathbf{r}_{\mathbf{2}} \frac{\left|\psi\left(\mathbf{r}_{1}\right)\right|^{2}\left|\psi\left(\mathbf{r}_{2}\right)\right|^{2}}{\left|\mathbf{r}_{1}-\mathbf{r}_{2}\right|} .
\end{aligned}
$$

Adapting the Gaussian trial function ansatz as before, the kinetic and external potential energy terms in the energy functional are easily calculated to yield $3 N \alpha / 2$ and $3 N / 8 \alpha$, respectively. The interaction energy term is calculated by going over to the center-of-mass coordinate system, yielding finally

$E_{I}=\gamma N^{2}\left(\frac{\alpha}{\pi}\right)^{3 / 2} 4 \pi \int_{0}^{\infty} r d r \mathrm{e}^{-\alpha r^{2}}=\frac{2 N^{2} \gamma}{\sqrt{\pi}} \alpha^{1 / 2}$.

The total variational energy is

$\frac{E}{N \hbar \omega}=\frac{3}{2} \alpha+\frac{3}{8 \alpha}+\frac{2 N \gamma}{\sqrt{\pi}} \alpha^{1 / 2}$.

Minimizing the energy with respect to $\alpha$, we get

$\frac{3}{2}-\frac{3}{8} \alpha^{-2}+\frac{N \gamma}{\sqrt{\pi}} \alpha^{-1 / 2}=0$.

Fig. 4 shows the $\alpha$ dependence of the total energy per particle in units of $\hbar \omega$ for $N=10^{4}$ atoms and $\gamma=1$. Fig. 5 also shows the same dependence but for the experimental parameters of Chen et al. [26] where the coupling parameter is $\gamma=10^{8}$. One can easily see the energy minimum in both curves despite the huge difference in the coupling strength values. Similarly to the Yukawa potential case, we obtain the cloud size for the bare Coulomb potential case as $\sim 2 \mathrm{~mm}$ where the trap frequency is approximately $10^{4} \mathrm{~Hz}$.

\section{Conclusion}

We have calculated the dependence of the size of a Bose condensed ionic cloud on the electron density, using ThomasFermi screening picture. This result may be used as a starting point for more accurate calculations of the plasma density, and

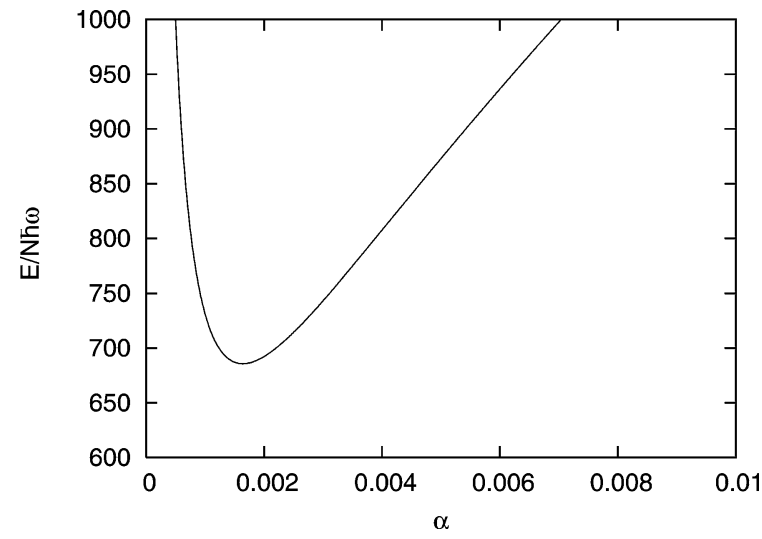

Fig. 4. Total energy per particle in units of $\hbar \omega$ as a function of variational parameter $\alpha$ for $N=10^{4}$ atoms for the bare Coulomb potential. The Coulomb coupling parameter is $\gamma=1$.

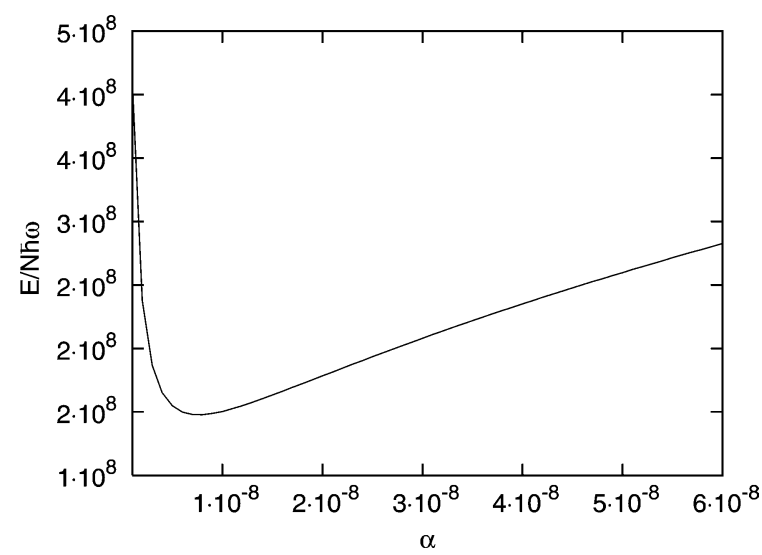

Fig. 5. Total energy per particle in units of $\hbar \omega$ as a function of variational parameter $\alpha$ for $N=10^{4}$ atoms for the bare Coulomb potential. The Coulomb coupling parameter is $\gamma=10^{8}$.

is directly relevant for the design of traps that can hold degenerate ultracold plasma. Our calculations show that to obtain stable ultracold plasma of similar sizes to current experiments, trapping frequencies must of the order of tens of $\mathrm{kHz}$.

\section{Acknowledgements}

M.Ö.O. thanks Aspen Center for Physics where part of this work was carried out. M.Ö.O. is supported by TUBA-GEBIP grant and TUBITAK-Kariyer grant No. 104T165. B.T. is supported by the Turkish Academy of Sciences (TUBA).

\section{References}

[1] M.H. Anderson, J.R. Ensher, M.R. Matthews, C.E. Wieman, E.A. Cornell, Science 269 (1995) 199;

K.B. Davis, M.-O. Mewes, M.R. Andrews, N.J. van Druten, D.S. Durfee, D.M. Kurn, W. Ketterle, Phys. Rev. Lett. 75 (1995) 3969.

[2] B. DeMarco, D.S. Jin, Science 285 (1999) 1703;

K.M. O'Hara, S.L. Hemmer, M.E. Gehm, S.R. Granade, J.E. Thomas, Science 298 (2002) 2179;

A.G. Truscott, K.E. Strecker, W.I. McAlexander, G.B. Partridge, R.G. Hulet, Science 291 (2001) 2570.

[3] C.A. Regal, M. Greiner, D.S. Jin, Phys. Rev. Lett. 92 (2004) 040403; 
K.E. Strecker, G.B. Partridge, R.G. Hulet, Phys. Rev. Lett. 91 (2003) 080406;

E. Hodby, S.T. Thompson, C.A. Regal, M. Greiner, A.C. Wilson, D.S. Jin, E.A. Cornell, C.E. Wieman, Phys. Rev. Lett. 94 (2005) 120402.

[4] T.C. Killian, S. Kulin, S.D. Bergeson, L.A. Orozco, C. Orzel, S.L. Rolston, Phys. Rev. Lett. 83 (1999) 4776.

[5] T.C. Killian, Nature 429 (2004) 815.

[6] C.E. Simien, Y.C. Chen, P. Gupta, S. Laha, Y.N. Martinez, P.G. Mickelson, S.B. Nagel, T.C. Killian, Phys. Rev. Lett. 92 (2004) 143001

[7] T. Pohl, T. Pattard, J.M. Rost, Phys. Rev. A 70 (2004) 033416.

[8] T.C. Killian, V.S. Ashoka, P. Gupta, S. Laha, S.B. Nagel, C.E. Simien, S. Kulin, S.L. Rolston, S.D. Bergeson, J. Phys. A 36 (2003) 6077.

[9] S.D. Bergeson, R.L. Spencer, Phys. Rev. E 67 (2003) 026414.

[10] A.N. Tkachev, S.I. Yakovlenko, JETP Lett. 73 (2001) 66.

[11] S. Kulin, T.C. Killian, S.D. Bergeson, S.L. Rolston, Phys. Rev. Lett. 85 (2000) 318.

[12] L.I. Minshikov, P.O. Fedichev, Zh. Eksp. Teor. Fiz. 108 (1995) 144.

[13] T.F. Gallagher, P. Pillet, M.P. Robinson, B. Laburthe-Tolra, M.W. Noel, J. Opt. Soc. Am. B 20 (2003) 1091.

[14] A.N. Tkachev, S.I. Yakovlenko, Quantum Electron. 31 (2001) 1084.

[15] T.C. Killian, M.J. Lim, S. Kulin, R. Dumke, S.D. Bergeson, S.L. Rolston, Phys. Rev. Lett. 86 (2001) 3759
[16] T. Pohl, T. Pattard, J.M. Rost, Phys. Rev. A 68 (2003) 010703.

[17] S.K. Dutta, D. Feldbaum, A. Walz-Flannigan, J.R. Guest, G. Raithel, Phys. Rev. Lett. 86 (2001) 3993.

[18] M.P. Robinson, B.L. Tolra, M.W. Noel, T.F. Gallagher, P. Pillet, Phys. Rev. Lett. 85 (2000) 4466.

[19] D. Ciampini, M. Anderlini, J.H. Müller, F. Fuso, O. Morsch, J.W. Thomsen, E. Arimondo, Phys. Rev. A 66 (2002) 043409.

[20] D.M. Stamper-Kurn, M.R. Andrews, A.P. Chikkatur, S. Inouye, H.-J. Miesner, J. Stenger, W. Ketterle, Phys. Rev. Lett. 80 (1998) 2027; S.R. Granade, M.E. Gehm, K.M. O'Hara, J.E. Thomas, Phys. Rev. Lett. 88 (2002) 120405.

[21] A.P. Gavrilyuk, I.V. Krasnov, N.Y. Shaparev, JETP Lett. 76 (2002) 423.

[22] S.A. Sekatskii, G. Dietler, Opt. Commun. 210 (2002) 251.

[23] T.B. Mitchell, J.J. Bollinger, D.H.E. Dubin, X.P. Huang, W.M. Itano, R.H. Baughman, Science 282 (1998) 1290.

[24] C. Kittel, Introduction to Solid State Physics, Wiley, New York, 1996.

[25] Y.E. Kim, A.L. Zubarev, Phys. Rev. A 64 (2001) 013603.

[26] Y.C. Chen, C.E. Simien, S. Laha, P. Gupta, Y.N. Martinez, P.G. Mickelson, S.B. Nagel, T.C. Killian, Phys. Rev. Lett. 93 (2004) 265003. 